\title{
Silicon-Germanium Multi-Quantum Wells for Extended Functionality and Lower Cost Integration
}

\author{
M. Cengiz Onbasli ${ }^{1}$, Alper Yesilyurt ${ }^{2}$, Hyun Yong Yu ${ }^{3}$, Ammar M. Nayfeh ${ }^{3}$, Ali K. Okyay ${ }^{2,4}$ \\ ${ }^{1}$ Department of Materials Science and Engineering, Massachusetts Institute of Technology, Boston, USA, MA 02139 \\ ${ }^{2}$ Department of Electrical and Electronics Engineering, Bilkent University, Ankara TR-06800, Turkey \\ ${ }^{3}$ Department of Electrical Engineering, Stanford University, Stanford, 94305, CA \\ ${ }^{4}$ UNAM, Institute of Materials Science and Nanotechnology, Bilkent University, Ankara, TR-06800, Turkey \\ e-mail:aokyay@ee.bilkent.edu.tr,onbasli@mit.edu
}

\begin{abstract}
Silicon-Germanium quantum wells were grown in p-i-n layers using a recently developed epitaxial technique. Nanostructural characterization (TEM, XPS, photoluminescence) indicates low-dislocation density, high quality films. Solar cells made of these layers have low leakage current.
\end{abstract}

Current state-of-the-art near infrared photodetectors are based on InGaAs [1], due to the material's (i) ease of integration with InGaAsP lasers for optical communication systems [2], (ii) high degree of tunability of the direct band gap energy and absorption [3] and (iii) low dark current using lattice matched wide bandgap materials [4]. The mature Silicon CMOS technology provides a lower cost platform while optoelectronics offers high performance and increased functionality. By integrating the two, one can meet the stringent market conditions of performance and functionality. Despite its performance advantage, current III-V technology suffers from (i) significant high cost of integration with Si CMOS [5], (ii) and high raw material costs [6,7]. Bulk Silicon is not suitable for photodetection in the telecommunication bands, since it cannot absorb strongly in the near infrared owing to its wide bulk band gap energy $(\sim 1.1 \mathrm{eV})$. For today's optoelectronic device applications, Germanium holds great promise with an absorption spectrum $\left(\mathrm{E}_{\mathrm{g}(\mathrm{direct})}=0.8 \mathrm{eV}, \mathrm{E}_{\mathrm{g} \text { (indirect) }}=0.66 \mathrm{eV}\right)$ spanning the telecommunication wavelengths at which $\mathrm{Si}$ is transparent. By growing $\mathrm{Ge}$ on $\mathrm{Si}$, it is also possible to integrate CMOS circuitry with optoelectronic components on single chip, which promises higher density and extended functionality [8].

Integration of Ge-based optoelectronic devices with Silicon demands high quality Ge layer growth. Ge growth on $\mathrm{Si}$, however, is encumbered by the large lattice mismatch $(4.2 \%)$ [6,7]. Nayfeh and coworkers recently introduced multiple hydrogen annealing heteroepitaxy (MHAH), technique achieving low defect density Ge layers on Si [8-10]. Here, we report the growth, optical and nanostructural characterization and device characterization of the MHAH-grown high quality thin Ge multi quantum well (MQW) films and the solar-cell characterization for devices made of these thin film MQW. The layers are doped in-situ forming an n-i-p structure with 10 pairs of quantum wells. MQWs provide (i) the ability to tune the material absorption by changing the bilayer thickness of each quantum well-barrier for operation at different wavelengths and (ii) the ability to exploit the quantum confined Stark effect (QCSE) for electroabsorption modulation [11]. In these applications, it is essential to control the layer thicknesses and material quality with a high precision. MHAH technique provides high materials quality as well as thickness precision. MQWs are one-dimensional periodic structures which can be utilized in various optoelectronic devices like near-infrared photodetectors, electroabsorption modulators based on the QCSE (which yields larger absorption difference than Franz-Keldysh effect for bulk), Ge-on-Si lasers. The on-chip integration of these devices using quantum wells is much easier and reproducible with respect to devices using other nanostructure topologies.
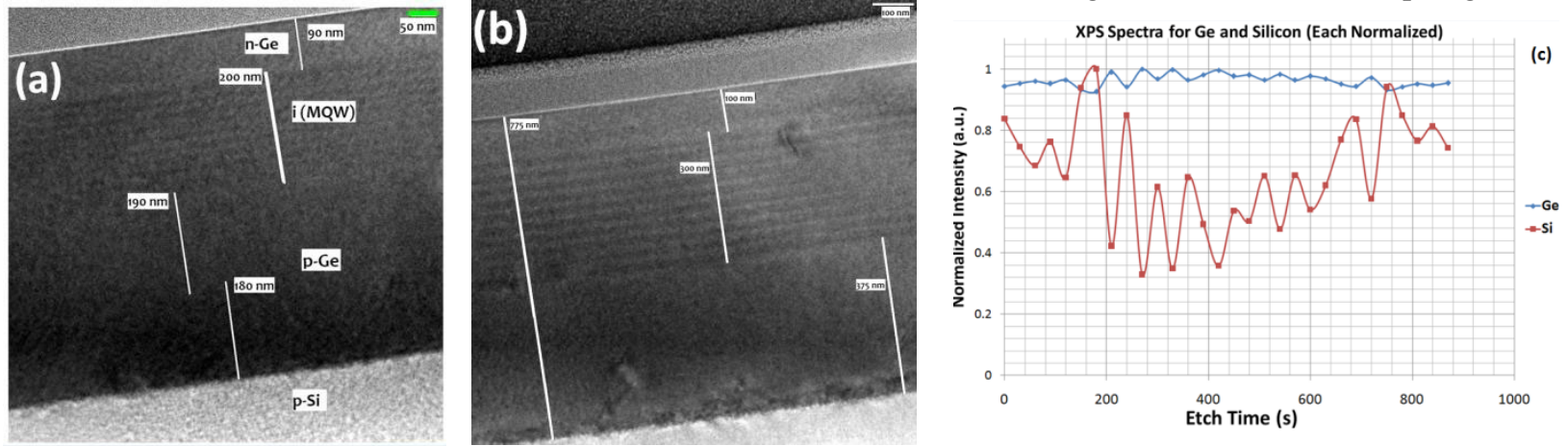

FIGURE 1. Cross sectional TEM images of the p-i-n SiGe MQW samples with well thickness $10 \mathrm{~nm}$ in (a), $15 \mathrm{~nm}$ in (b). (c) Normalized XPS profile showing material composition

Silicon wafers with (100) surface orientation and p-type doping were first cleaned using standard RCA clean with HF-last step. The wafers were immediately loaded to an Applied Materials reduced pressure epitaxial reactor. 
As the first step of all the growths, the wafers were baked in hydrogen ambient at $950^{\circ} \mathrm{C}$ for 5 minutes. Then an ultra-thin seed layer at $700^{\circ} \mathrm{C}$ was grown to ensure an ultra-clean surface to start high quality Ge growth. Temperature was then reduced to $400^{\circ} \mathrm{C}$ and a 180 -nm-thick p-type Ge buffer layer was grown. Subsequently, wafer temperature was ramped up to $825^{\circ} \mathrm{C}$ and baked for 20 minutes in hydrogen ambient. Before further Ge growth, this step is needed to facilitate surface reconstruction. The presence of hydrogen extends surface adatom diffusion length, reducing surface roughness. Dislocations glide and annihilate during this anneal. The temperature was set to $400^{\circ} \mathrm{C}$ once again and the previous growth and anneal steps were repeated. Further growth was performed to obtain undoped MQW regions on Ge virtual substrates, which were obtained by the dual by grow-anneal cycles. Alternating Ge well region and SiGe barrier regions were grown at $400^{\circ} \mathrm{C}$. Intermediate annealing was avoided for preventing (i) any interdiffusion of $\mathrm{Si}$ and $\mathrm{Ge}$ and (ii) diffusion of doping species from underlying highly doped regions. Finally, a 100-nm-thick n-type Ge layer was grown as a cap and provides ohmic contact to the n-i-p device.

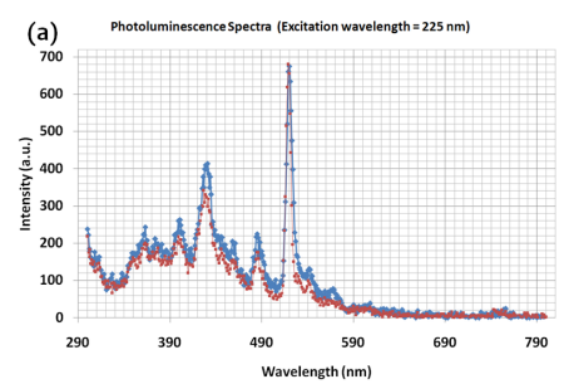

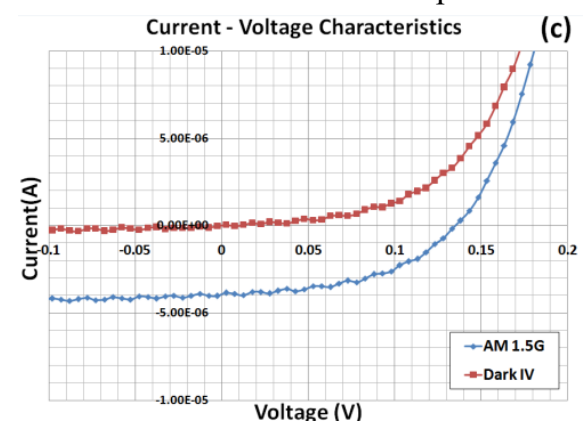

FIGURE 2. (a) Photoluminescence of samples in Fig.1(a) and (b). (b) Device SEM. (c) I-V characteristics of a p-i-n device.

Transmission electron micrographs (TEM), as seen in Figure 1(a) and (b), reveal that quantum wells have formed with low dislocation density inside the n-i-p layers. X-Ray photoelectron spectroscopy (XPS) was used to investigate the concentrations of $\mathrm{C}, \mathrm{O}, \mathrm{Ge}$ and $\mathrm{Si}$ as a function of depth. The layers were etched in-situ by a secondary beam and then the scattered energy spectrum was recorded periodically. About $6 \mathrm{~nm}$ was etched for each 10 -second intervals and then scattered spectrum was recorded, as plotted in Fig.1(c). The undulating behavior of silicon concentration between etch-cycles 200 and 750 seconds is due to the barrier and well regions. Silicon content inside the wells of the intrinsic layers are minimal when there is Germanium rich SiGe alloy. Leakage or contamination was below the detectable limit in the XPS spectra. Photoluminescence spectrum was measured for different quantum well samples and to probe the electronic structure by light excitation at $\lambda=225 \mathrm{~nm}$. The layers have the sharp luminescence peak at $520 \mathrm{~nm}$, as in Fig. 2(a). Then, MQW n-i-p layers were used for fabrication of circular MESA structures for detector and solar cell mode operation. Photolithography and reactive ion etching was used for defining the MESAs. For surface passivation and antireflection, Silicon nitride was deposited on the devices by PECVD. Thermally evaporated Al formed the top and bottom electrodes. Reverse leakage current of 22.2 $\mathrm{nA}$ at $-0.1 \mathrm{~V}$ bias was measured for the devices with $20 \mu \mathrm{m}$ MESA diameter. Under AM1.5G solar simulator illumination, short circuit current of $3.9 \mu \mathrm{A}$ and open circuit voltage of $137 \mathrm{mV}$ was measured for $150 \mu \mathrm{m}$ diameter device.

In this study, we investigated the materials and device quality of the SiGe MQW layers grown with a previously developed technique called MHAH. MHAH enables the high quality growth of Ge rich MQW on Silicon substrates. The n-i-p films have been characterized to have high materials quality (i.e. low dislocation density). Solar cells made of these layers have low leakage current and high photocurrent. This technology is very promising to integrate optoelectronic devices with Si on CMOS platform.

\section{References}

[1] Thorlabs Catalogue version 20. Thorlabs. 2010. June 24, 2010. $<$ http://www.thorlabs.com/images/Catalog/V20/V20_7_LightAnalysis.pdf >

[2] Sasaki et. al., Journal of Crystal Growth, 132, 3-4, 2 September 1993, Pages 435-443

[3] Saleh, B. E. A., Teich, C. M., "Fundamentals of Photonics $2^{\text {nd }}$ Edition", 2007, Wiley-Interscience

[4] B. Onat, Proc. of SPIE Vol. 6542, 65420L, (2007)

[5] Cohen D., "How Long Will it Last?". New Scientist 194 (2605): 38-39. May 26, 2007

[6] Ali K. Okyay, "Si Ge Photodetection Technologies for Integrated Optoelectronics", PhD Thesis, Stanford University, 2007.

[7] Ammar M. Nayfeh, "Heteroepitaxial Growth of Relaxed Germanium on Silicon", Ph.D Thesis, Stanford University, 2006.

[8] O. Fidaner et. al., Photonics Technology Letters, IEEE, vol.19, no.20, pp.1631-1633, Oct.15, 2007

[9] A. Nayfeh et. al. Appl. Phys. Lett. 85, 2815 (2004)

[10] A. K. Okyay. et. al., Opt. Lett. 31, 2565-2567 (2006)

[11] Kuo et. al., Nature 437, 1334-1336 (27 October 2005) 